\title{
Implications of doping on microstructure, processing, and thermoelectric performance: The case of $\mathrm{PbSe}$
}

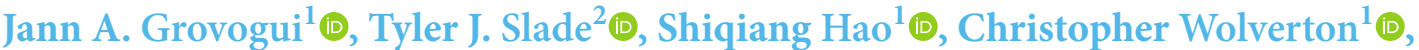 \\ Mercouri G. Kanatzidis ${ }^{2}$, Vinayak P. Dravid ${ }^{1, a)}(0)$ \\ ${ }^{1}$ Department of Materials Science and Engineering, Northwestern University, Evanston, IL 60208, USA \\ ${ }^{2}$ Department of Chemistry, Northwestern University, Evanston, IL 60208, USA \\ a) Address all correspondence to this author. e-mail: v-dravid@northwestern.edu \\ Received: 5 November 2020; accepted: 9 February 2021; Published online: 9 April 2021
}

In this work, we highlight the often-overlooked effects of doping on the microstructure and performance of bulk thermoelectric materials to offer a broader perspective on how dopants interact with their parent material. Using $\mathrm{PbSe}$ doped with $\mathrm{Na}, \mathrm{Ag}$, and $\mathrm{K}$ as a model material system, we combine original computational, experimental, and microscopy data with established trends in material behavior, to provide an in-depth discussion of the relationship between dopants, processing, and microstructure, and their effects on thermoelectric efficiency and thermal stability. Notable observations include differences in the microstructure and mass loss of thermally treated samples of $\mathrm{Na}$ - and $\mathrm{Ag}$-doped $\mathrm{PbSe}$, as well as findings that $\mathrm{Na}$ and $\mathrm{K}$ cations exist predominantly as substitutional point defects while $\mathrm{Ag}$ also occupies interstitial sites and exhibits lower solubility. We discuss how these differences in point defect populations are known to affect a dopants' ability to alter carrier concentration and how they may affect the mechanical properties of $\mathrm{PbSe}$ during processing.

\section{Introduction}

Thermoelectric devices are an attractive technology for a variety of applications such as waste-heat energy conversion, space flight, and electronic cooling because of their ability to directly convert heat into electricity (and vice versa). Another attractive feature of thermoelectrics is their lack of moving parts, which allows them to function for decades without degradation from mechanical wear and tear [1-4]. An incredible display of longevity is the fact that the Radioisotope Thermoelectric Generators (RTGs) used to power Voyager 1 have been functioning since the deep space probe was launched by NASA in the late 1970s [2, 5]. Although thermoelectric devices have proven to be successful in space, for most terrestrial applications, materials need to be more efficient to become economically feasible [4].

The efficiency of a thermoelectric material is often described using the material figure of merit, which is expressed as $z T=\left(\sigma S^{2}\right) T / \kappa_{t o t}$, where $\sigma$ is electrical conductivity, $S$ is the Seebeck coefficient, $\kappa_{\text {tot }}$ is the total thermal conductivity, and $T$ is absolute temperature. To improve efficiency, the goal is to increase the electrical properties, defined by the power factor $\left(\sigma S^{2}\right)$, while simultaneously decreasing the total thermal conductivity. In practice, the individual manipulation of electrical and thermal properties presents a challenge because these properties are intricately linked. As a result, thermal and electrical properties usually increase and decrease in unison, yielding little to no net change in $z T$. A major challenge in the field of thermoelectrics is to decouple these properties to obtain an overall enhancement in $z T$, thereby increasing efficiency [1, 2, 6-8].

Semiconductors are of particular interest in thermoelectricity because they have intrinsically high Seebeck coefficients compared to metals. With the addition of small amounts of impurity elements (dopants) to increase the carrier concentration, the electrical conductivity (and subsequently the power factor) of degenerately doped semiconductors becomes highly favorable for thermoelectric applications [8]. In addition to enhancing the power factor, point defects created by doping lead to additional scattering mechanisms for phonons that decrease thermal conductivity [9]. Owing to the ability to tune the Fermi level (carrier concentration) while simultaneously enhancing phonon scattering, extrinsic doping is almost always used to optimize 
the performance of semiconducting thermoelectric materials. Although increasing the carrier concentration and adding point defects are vitally important to increasing the efficiency of thermoelectrics, energy conversion efficiency is not the only metric that determines the potential effectiveness of a thermoelectric device. The use of thermoelectrics in RTGs makes it clear that the longevity of thermoelectric materials is also an essential quality, so for practical application, optimal performance for thermoelectrics should take into consideration both a material's efficiency as well as its durability (or resistance to degradation over time). For example, when specifically considering doping, some dopants such as Ag may provide better high-temperature stability to lead chalcogenides than either $\mathrm{Na}$ or $\mathrm{K}$ because of the high diffusion rate of alkali metals [10]. However to date, the broader impacts of doping outside of its contributions to transport properties have been sparsely considered and not thoroughly investigated.

To address these concerns, we elected to study $\mathrm{PbSe}$ as a model system because it is already a commonly studied thermoelectric material. $\mathrm{PbSe}$ is a promising analogue to $\mathrm{PbTe}$ (one of the best performing intermediate temperature thermoelectrics) because of its similar crystal and electronic band structure, and because of the relative Earth abundance of Se compared to Te [11-13]. PbSe has a combination of light and heavy valence bands which make it favorable for p-type doping, and therefore this study focuses on the direct comparison of different p-type dopants $[10,11,14,15]$. Specifically, this report investigates $\mathrm{PbSe}$ doped with $\mathrm{Na}, \mathrm{K}$, and $\mathrm{Ag}$ and uses defect energy calculations to reveal the nature of the underlying point defects present in doped $\mathrm{PbSe}$ and the relationship between dopant species selection and the resultant doping efficiency, microstructure, and thermal stability of PbSe. Despite doping concentrations of $2 \%$ or less, we discuss how the choice of dopant, combined with processing, may impact both the grain size and low-angle grain boundary population in PbSe. Furthermore, we seek to demonstrate how these factors impact the thermal stability of samples annealed at high temperatures, normally considered within the operating range of PbSe thermoelectrics. This work emphasizes how developing a keen understanding of the relationship between dopant species and microstructure is critically needed to help inform synthetic processes which can lead to the fabrication of thermoelectrics optimized for both efficiency and thermal stability.

\section{Results}

\section{Microstructure}

We first prepared samples of PbSe doped with nominally $2 \%$ $\mathrm{Na}, \mathrm{K}$, or Ag. The initial samples in this investigation underwent the same synthesis and post-synthetic processes, were cut from the same area of their respective pellets, and were imaged in the same viewing direction relative to the pressing direction. Yet upon investigation of their microstructures, we surprisingly observe stark differences in both the grain size and the population of low-angle grain boundaries (LAGBs). We emphasize that the only intentional difference between the samples is the identity of the p-type dopant: pure PbSe (no dopant), PbSe-2\%Na, PbSe-2\%K, and PbSe-2\%Ag. Figure 1 reveals both the grain structure and grain boundary angle distribution in the $\mathrm{PbSe}$ samples prior to annealing. Mixed forward scattered electron (FSE) images displaying orientation contrast show the consistent difference in grain size between $\mathrm{Na}$ - and Ag-doped PbSe over a larger field of view in SI Fig. $\mathrm{S} 1$. It should be noted that after annealing there appears to
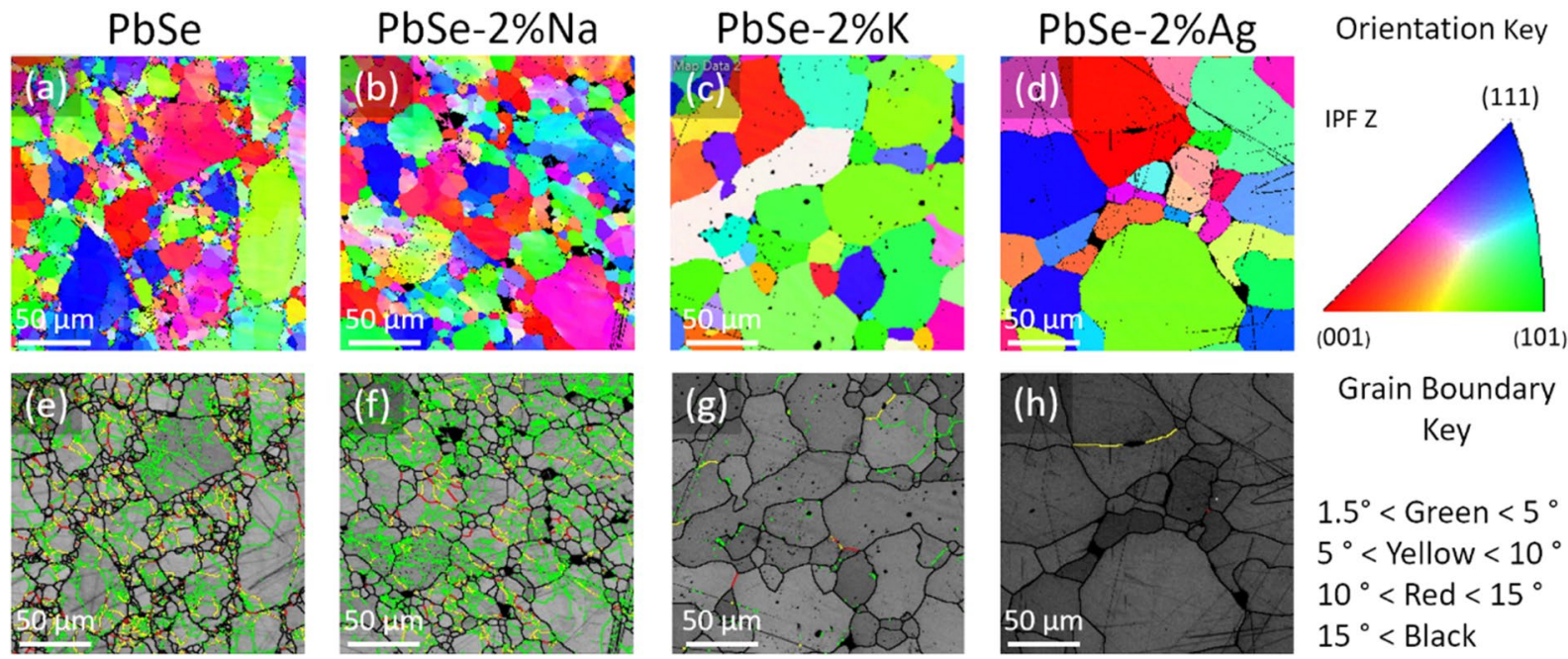

Figure 1: Unannealed, single sieved PbSe with varying dopant species. EBSD (a-d) Inverse Pole Figure orientation maps highlighting the grain size and (e-h) Band Contrast maps with color coded LAGBs. 
be grain growth in some samples (see SI Fig. S2); however, the difference in grain sizes between samples remains large throughout the thermal treatment. To compare the thermal stability of different samples, the grain size of each sample should be as consistent as possible at high temperatures to reduce the amount that microstructural variations affect subsequent mass loss results. Obtaining a uniform final grain size in powder processed materials is not trivial, and in this investigation, we did not seek to address or solve all the imperfections associated with our powder processing method, Spark Plasma Sintering (SPS). Our goal was to develop an extra step that may be able to help improve microstructural uniformity so that we could make more accurate comparisons between the thermal stability of differently doped samples with minimal influence from microstructural effects.

Sieving is used to control the maximum grain size of sintered materials by limiting the size of the precursor powder particles used in the solidification process. In other words, if the grain size of the precursor material is much larger than the powder size, then the powder size will determine the minimum grain size in the sintered material. Alternatively, if the grain size of the precursor material is smaller than the powder size (i.e., the powder particles are multi-grain), then sieving will not be able to limit the minimum grain size. In bulk thermoelectric materials, the precursor material is usually an ingot solidified from melt, and therefore likely has some distribution of grain sizes. This means that sieving can control the maximum grain size, but it may not be able to eliminate all small grains in the final sintered materials, unless the powder size is exceedingly small. It has already been shown that crushing, sieving, and solidification are well-established methods of producing bulk micro- and nanostructured thermoelectric materials with controlled grain sizes [16-18], and our prior investigations with analogous compounds show that the grains in our slowly cooled lead chalcogenide ingots can be upwards of millimeters in size [19, 20], while our powder size is targeted to be $10 \mathrm{~s}$ of micrometers. Therefore, although we could not eliminate the presence of all small grains, we suspected that a tighter control over the size of our precursor powder would yield some improvement in the uniformity of the grain sizes between PbSe samples doped with different dopants.

To provide more confidence in the uniformity of our samples, we introduced a processing step referred to here as "double sieving". During typical sieving, crushed powder is passed through a single mesh, which only determines maximum powder size, but does not limit the minimum powder size. In the case of $\mathrm{PbSe}-2 \% \mathrm{Na}$, even though the mesh size was $70 \mu \mathrm{m}$ the sample has many grains on the order of 10-20 $\mu \mathrm{m}$. In the new process, we control maximum and minimum powder size by sieving the powder through a $150 \mu \mathrm{m}$ mesh and then sieving the resultant powder through a $53 \mu \mathrm{m}$ mesh. We then SPS the intermediate sized powder (that which was sifted through the first mesh but did not get filtered through the second mesh) to create our new, more uniform samples, which we refer to as "large-grain" in this text because of our attempt to filter out small powder sizes.

Electron Backscattered Diffraction (EBSD) of the largegrained $\mathrm{PbSe}-2 \% \mathrm{Na}$ sample reveals that grain sizes were somewhat comparable to Ag-doped samples. However, the microstructure of the large-grained Na-doped material still differs from that of the Ag-doped PbSe because of the large amount of low-angle grain boundaries (LAGBs) that are present in $\mathrm{Na}$ doped PbSe. The network of LAGBs is displayed in the EBSD band contrast map in Fig. 2d, which highlights LAGBs in green, yellow, and red, while those with misorientation angles above $15^{\circ}$, also known as high-angle grain boundaries (HAGBs), are highlighted in black [21]. SI Fig. S3 uses EBSD maps to illustrate a direct comparison of the grain structures of a single and double sieved $\mathrm{Na}$-doped sample, and the data used to create these maps were used to calculate average grain sizes of $22.3 \mu \mathrm{m}^{2}$ and $45.4 \mu \mathrm{m}^{2}$ for single and double sieved samples, respectively.

\section{High-temperature mass loss}

To document the thermal stability of doped PbSe samples, we first exposed highly polished $\mathrm{Na}$-doped $\mathrm{PbSe}$ to temperatures of $600-650^{\circ} \mathrm{C}$ for $48 \mathrm{~h}$. We then used SEM to analyze the surfaces after annealing to gain a qualitative understanding of the extent of mass loss. Figure 3 shows a combination of surface pores, rough surface grains, and plateau/tree-like structures. These features formed because of material removal from the surface of the sample, which had originally been smooth and polished for EBSD using the steps described in the methods section. From this figure, we can see that most of the new pores are located at what appear to be grain boundaries, which agrees with the fact that grain boundaries sublimate at a higher rate than the defect-free material [22-24]. Figure 2a further illustrates the strong relationship between grain boundaries and mass loss by using EBSD to highlight pores preferentially forming at HAGBs. The pores represented by unindexed pixels in the EBSD maps in Fig. 2a-d can also be observed directly in the secondary electron images in the SI in Fig. S4. Energy Dispersive Spectroscopy (EDS) was performed simultaneously with EBSD scans of unannealed samples (SI Fig. S5) and reveals that there is no chemical segregation at grain boundaries within the detection limits of SEM-EDS. This does not completely rule out the possibility of very slight variations in composition at grain boundaries. However, whether the increase in mass loss at grain boundaries is due to slight compositional variation or is a function of the elevated defect energy of grain boundaries (due to their structural imperfections), SEM imaging and EBSD are able to qualitatively illustrate that the grain boundaries are more susceptible to thermal instability than the defect-free bulk. 


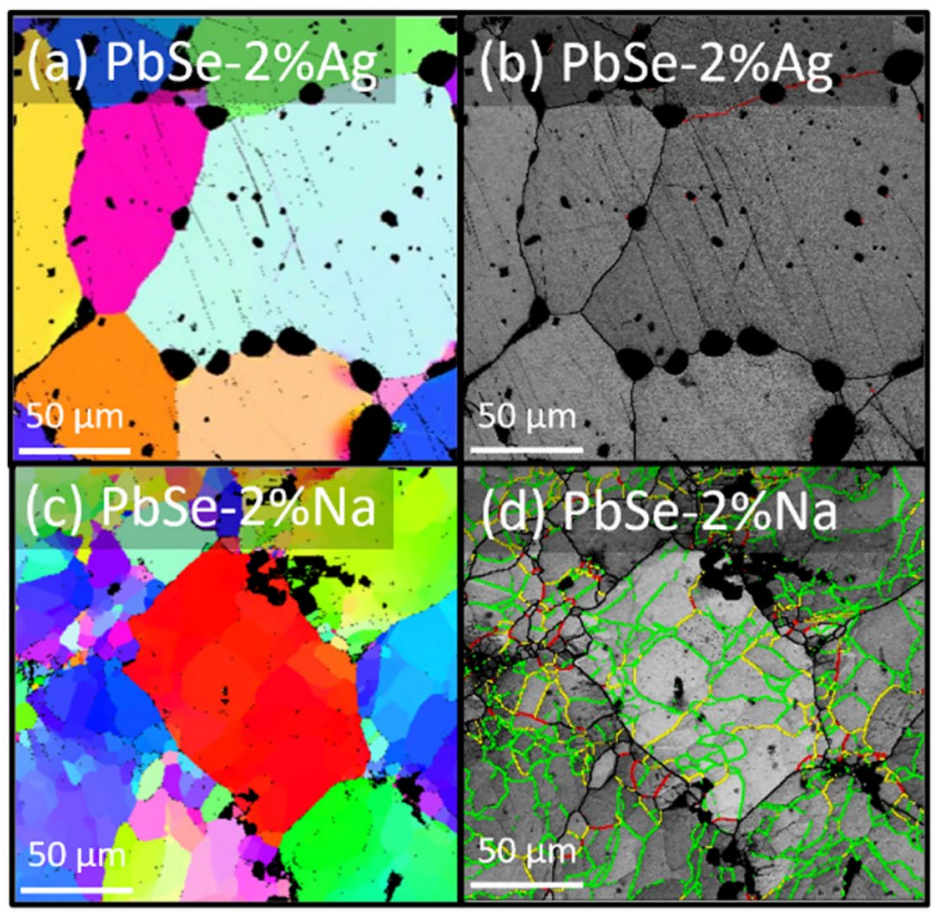

\section{Orientation Key}

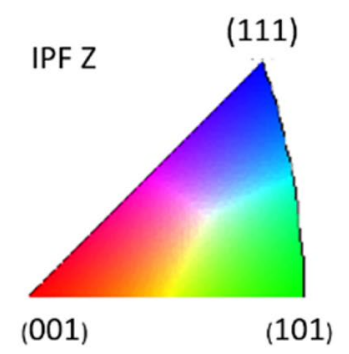

\section{Grain Boundary \\ Key}

$1.5^{\circ}<$ Green $<5^{\circ}$

$5^{\circ}<$ Yellow $<10^{\circ}$

$10^{\circ}<\operatorname{Red}<15^{\circ}$

$15^{\circ}<$ Black

Figure 2: $(a, b)$ Annealed PbSe-2\%Ag with pores forming at grain boundaries. (c, d) Annealed large-grained PbSe-2\%Na (processed using the "double sieve" method) with pores predominantly forming at HAGBs.

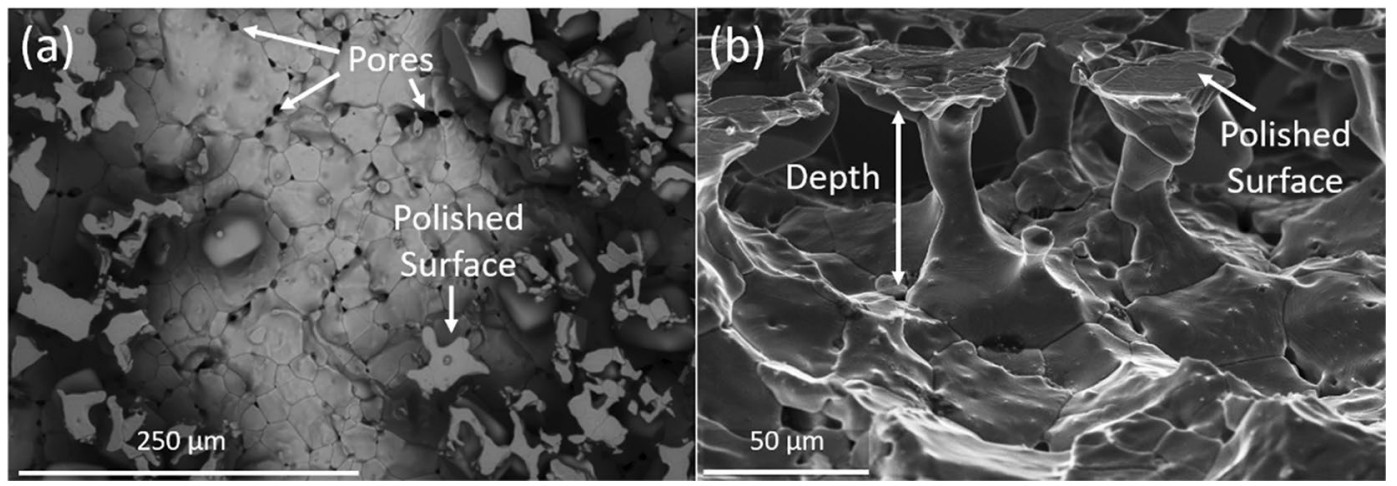

Figure 3: Secondary electron images of (a) the top and (b) angled side view of the surface features of $\mathrm{Na}$-doped PbSe samples that had been annealed between $600^{\circ} \mathrm{C}$ and $650^{\circ} \mathrm{C}$ for $48 \mathrm{~h}$. Annealing resulted in the sublimation of the surface of the material and subsequent degradation of material below the initial surface as well. The flat features are the remnants of the original sample surface that had been polished for EBSD prior to annealing, while the rough textured surfaces and pores are portions of the sample that have been exposed due to the removal of the original surface material.

To obtain more quantitative results, the mass of PbSe samples was measured before and after annealing. The first set of mass loss experiments were performed on the same set of samples seen in Fig. 1 (pure PbSe and PbSe doped with 2\% Na, K, and $\mathrm{Ag}$ ), which were all synthesized using a single-sieve powder separation method. The results of the first mass loss experiment can be seen in Fig. 4a. Because grain structure was less uniform between PbSe samples doped with different elements, and because we know that grain boundaries increase pore formation (and presumably mass loss), further experiments were performed to better understand the effects of mass loss with a reduced influence from the microstructure. Additionally, because pure $\mathrm{PbSe}$ exhibited the lowest mass loss in the first set of experiments, we wanted to test the effects of dopant concentration on thermal stability. For this reason, a second set of quantitative mass loss experiments were performed on large-grain $\mathrm{PbSe}$ $2 \% \mathrm{Na}$ and large-grain PbSe- $0.4 \% \mathrm{Na}$. Data displayed in Fig. $4 \mathrm{~b}$ show that reducing the dopant concentration from $2 \%$ to $0.4 \%$ in Na-doped samples reduces mass loss from an average of $5 \%$ to $2 \%$. Furthermore, with standardization of grain size, the mass loss of $\mathrm{PbSe}-0.4 \% \mathrm{Na}$ becomes comparable to the mass lost from $\mathrm{PbSe}-2 \% \mathrm{Ag}$. 
Mass Loss Based on Dopant Species (No Microstructural Standardization)

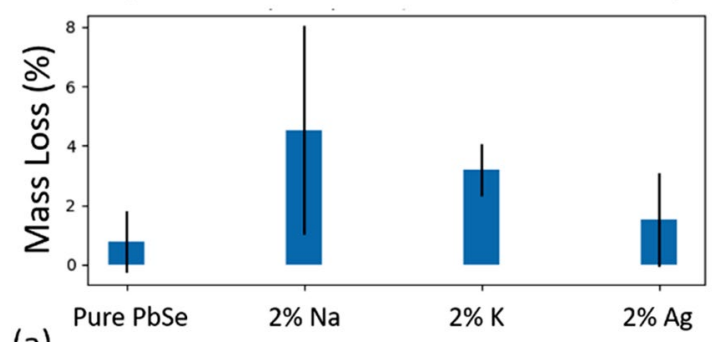

(a)

Mass Loss Based on Dopant Species and Carrier Concentration

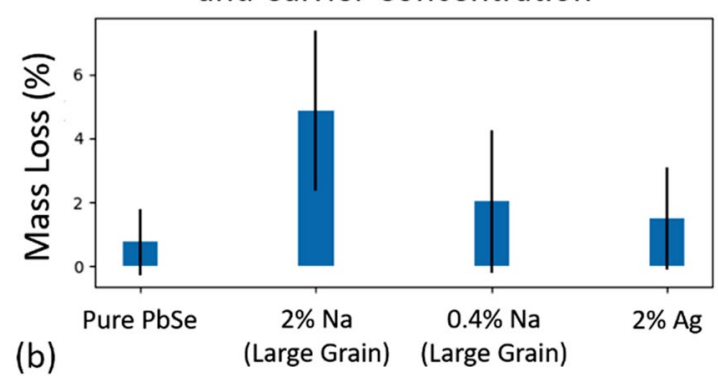

Figure 4: Mass Loss due to annealing PbSe at $650^{\circ} \mathrm{C}$ for $48 \mathrm{~h}$. (a) Initial mass loss experiments comparing mass loss of $\mathrm{PbSe}$ with equal amounts of different dopant species prior to microstructural standardization. (b) Na-doped samples were synthesized using "double sieving" and with reduced carrier concentration to measure the effects of dopant concentration and dopant species on samples with comparable grain sizes.

\section{DFT defect energy calculations}

To provide insight into the various effects that dopants have on the microstructure and performance of $\mathrm{PbSe}$, it is important to understand how defects interact with the host material. Therefore, Density Functional Theory (DFT) was used to calculate point defect energies in PbSe doped with different elements. Figure 5 plots the defect energies in the three doped PbSe samples as a function of Fermi level $\left(E_{\mathrm{f}}\right)$, where $E_{\mathrm{f}}=0 \mathrm{eV}$ corresponds to the valence-band maximum, and a decrease in $E_{\mathrm{F}}$ indicates greater p-type doping. In these plots, we defined the defects using Kröger-Vink notation with effective charge defined by

$$
q_{e}=z_{d}-z_{s}
$$

where $q_{\mathrm{e}}$ is the effective charge, $z_{\mathrm{d}}$ is the charge of the defect, and $z_{\mathrm{s}}$ is the charge of the original site [25]. Using this notation, a cation substitution that results in p-type doping would be written as $X_{\mathrm{Pb}}^{\prime}$, where $X$ is the dopant species. In this case, $\mathrm{Pb}^{2+}$ is the original cation and gives $z_{\mathrm{s}}$ a value of 2 , while the dopants $\left(\mathrm{Na}^{+}\right.$, $\mathrm{K}^{+}$, or $\mathrm{Ag}^{+}$) give $z_{\mathrm{d}}$ a value of 1 . According to Eq. (2), the effective charge of the defect is -1 , and the Kröger-Vink notation for the cation substitutional defect is $X_{\mathrm{Pb}}^{\prime}$.

In all three doped materials, cation substitutions are the lowest energy defects at the onset of doping, and therefore are the dominant defect species, until $\mathrm{E}_{\mathrm{F}}$ becomes sufficiently negative and a crossover between defect energies occurs. In $\mathrm{Na}$ - and $\mathrm{K}$-doped $\mathrm{PbSe}$, this crossover is due to the presence of Se vacancies $\left(V_{\mathrm{Se}}^{2 \cdot}\right)$, while in Ag-doped $\mathrm{PbSe}$ the crossover is due to the presence of $\mathrm{Ag}^{+}$interstitials $\left(\mathrm{Ag}_{i}^{*}\right)$. Additionally, the crossover in Ag-doped PbSe occurs at a higher Fermi level than in the Naand K-doped materials, which indicates that cation substitutions will stop being the dominant defect at lower levels of doping when using Ag as a p-type dopant compared to either $\mathrm{Na}$ or $\mathrm{K}$.

\section{Discussion}

\section{Point defects and doping efficiency}

DFT reveals that changing the dopant species in PbSe can alter the types of point defects that are present in the material. The
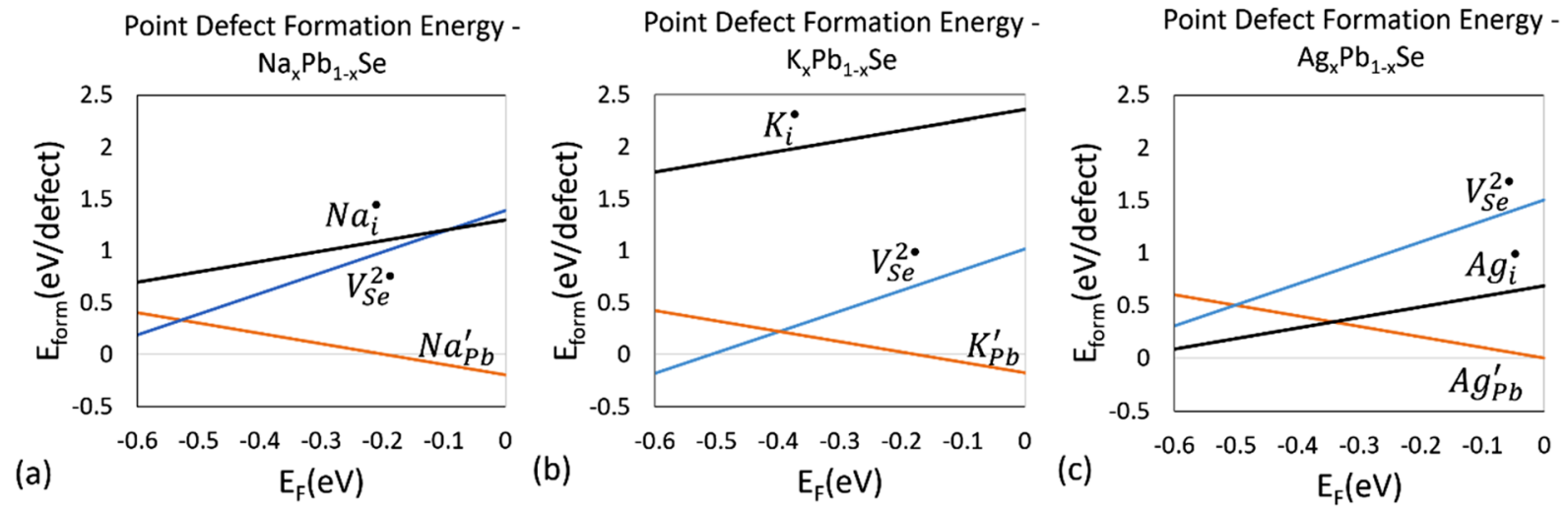

Figure 5: DFT calculations of defect energies in PbSe doped using different dopants. A Fermi level ( $\mathrm{E}_{\mathrm{F}}$ ) of 0 eV corresponds to the top of the valence band, and as p-type doping increases, $\mathrm{E}_{\mathrm{F}}$ becomes more negative. The primary point defect in the system is that which has the lowest defect energy at a given $\mathrm{E}_{\mathrm{F}}$. The crossover point shows the level of doping at which compensating defects begin to dominate. Ag interstitial cations (denoted by $A g_{i}^{\bullet}$ ) are the primary compensating defect in Ag-doped PbSe and their crossover point occurs at a higher Fermi level than the Se vacancy $\left(V_{S e}^{2 \bullet}\right)$ crossover points in any of the three material systems calculated. 
calculations are further supported by the fact that the types of defects predicted by our DFT study align well with the established doping efficiency of $\mathrm{Na}, \mathrm{K}$, and $\mathrm{Ag}$ in PbSe $[10,11,15]$. Doping efficiency is the relationship between the quantity of dopant added and the resulting number of charge carriers, which is critical to increasing carrier concentration to its optimal level. There is not a perfect relationship between the quantity of dopant that is added and the resulting number of carriers. This is because, in addition to the desired substitutional point defects caused by doping, additional defects of opposite charge (compensating defects) also form. In $\mathrm{Na}$ - and $\mathrm{K}$-doped PbSe, the compensating defects manifest as Se vacancies, while in Agdoped PbSe these defects manifest as cations sitting at interstitial sites, both of which contribute electrons to the system rather than holes. Ultimately compensating defects will limit the maximum carrier concentration by pinning the Fermi level at the energy crossover, because the compensating defects become the dominant defect and p-type dopants cannot be added without the creation of defects that add electrons to the system [26-28].

The DFT results show that the energy crossover in Ag-doped $\mathrm{PbSe}$ occurs at a higher $\mathrm{E}_{\mathrm{F}}$ than in $\mathrm{Na}$ - and $\mathrm{K}$-doped $\mathrm{PbSe}$, indicating that compensating defects should occur at lower levels of doping, lowering the p-type doping efficiency. As illustrated in Fig. 6, the p-type doping efficiencies predicted by our calculations are consistent with the established behaviors of doped $\mathrm{PbSe}$, where Ag-doped $\mathrm{PbSe}$ shows a strong deviation from perfect doping efficiency (and a low maximum p-type carrier concentration) compared to its $\mathrm{Na}$ - and $\mathrm{K}$-doped counterparts $[10,11,15]$. Silver's amphoteric behavior, which results in its donation of both holes and electrons to lead chalcogenides, has previously been linked to a combination of interstitial point defects and precipitates in $\mathrm{PbTe}[31,32]$. In PbSe, precipitates

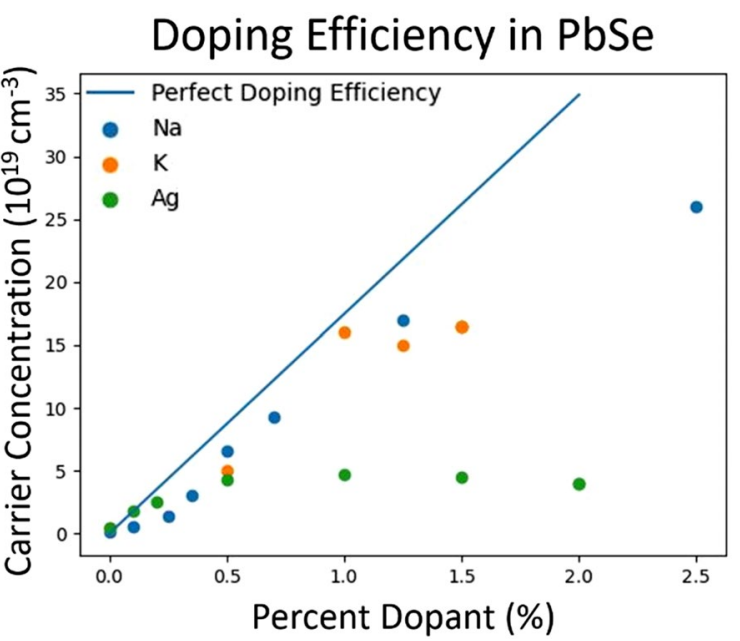

Figure 6: A plot of data collected from literature illustrating the difference in doping efficiency of Ag-doping [10], Na-doping [11], and K-doping [15] in the PbSe thermoelectric system. have been predicted to form when the concentration of $\mathrm{Ag}$ is between 0.5 and 1\% [10], although our Powder X-Ray Diffraction (PXRD) results indicate that all of our samples were single phase (see SI Fig. S6). However, it should be noted that because Ag-doping can result in the creation of interstitial point defects, there is the possibility that Ag could experience lower solubility in heavily doped $\mathrm{PbSe}$ due to increased lattice strain because of the size of the $\mathrm{Ag}^{+}$cation. In summary, our DFT results show that the type of dopant used in a material can alter the underlying point defects. We were able to further validate the specific defects predicted in each doped PbSe system by comparing the predicted defects to the established changes in doping efficiency that these dopants produce. With increased confidence in the type of point defects present in each doped PbSe system, the following sections discuss the relationships between dopant species, microstructure, and thermal stability.

\section{Low-angle grain boundaries}

LAGBs are of interest because they are formed by arrays of dislocations, which are thought to be ideal for scattering intermediate wavelength phonons. This has made dislocations the focus of multiple studies aiming to decrease lattice thermal conductivity in thermoelectric materials [29-31]. Therefore, the formation mechanism of LAGBs is important. Recrystallization (the formation of new strain free grains) is driven by the reduction of stored energy in a material [32]. If LAGBs and subgrains form as a result of recrystallization, then this may reduce phonon scattering in thermoelectric materials. In terms of thermal stability, LAGBs generally exhibit lower defect energies than HAGBs, making them less susceptible to high-temperature mass loss via sublimation [33]. The fact that LAGBs and subgrain formation can influence material volatility as well thermal transport makes controlling these defects important to thermoelectric performance and long-term device operation. Our investigation reveals a definitive change in the prevalence of LAGBs that is dependent on the type of dopant being used. In this section, we suggest several mechanisms that may contribute to the specific trend in decreasing LAGB density as dopants change from $\mathrm{Na}$ to $\mathrm{K}$ to $\mathrm{Ag}$ in $\mathrm{PbSe}$.

For dislocation stacking (and therefore LAGB formation) to occur, dislocations need to be mobile. In ceramics this happens at high homologous temperatures and pressures where dislocation creep can occur, meaning that stress exponents exceed 3 [34-37]. Past studies have shown how creep affects chalcogenide-based thermoelectrics during high-temperature operation, but extreme conditions can be present during processing as well [38-40]. During our synthesis and postsynthetic processing, the only step that exposes materials simultaneously to high temperatures and pressures is SPS. Specifically, samples are exposed to pressures of $40 \mathrm{MPa}$ and 
temperatures as high as $550{ }^{\circ} \mathrm{C}\left(0.61 \mathrm{~T}_{\mathrm{m}}\right.$ of $\left.\mathrm{PbSe}\right)$. These conditions are presumably adequate to enable dislocation motion in $\mathrm{PbSe}$, as they are in excess of the conditions that produce a stress exponent of $\mathrm{n}=4.4$ in its structural analogue $\mathrm{PbTe}$ at $400{ }^{\circ} \mathrm{C}\left(0.56 \mathrm{~T}_{\mathrm{m}}\right.$ of $\left.\mathrm{PbTe}\right)$ and pressures ranging from 7 to $35 \mathrm{MPa}[40]$.

Because PbSe is exposed to creep conditions during SPS, it will either act as a Class I material (with stress exponent close to $n=5$ ), in which subgrain formation occurs primarily by dislocation climb, or a Class II material (with stress exponent closer to $n=3$ ) in which dislocation glide dominates and subgrains form with more difficulty [34-37]. Generally speaking, non-metals with $r_{\text {anion }} / r_{\text {cation }}<2$ exhibit Class I behavior, and so we would predict that $\mathrm{PbSe}$ (much like $\mathrm{PbTe}$ ) would have a stress exponent closer to $n=5$ [41]. Additionally, it has been shown in a variety of ionic rocksalt compounds that pure ceramics (such as $\mathrm{NaCl}, \mathrm{KCl}, \mathrm{KBr}$, and $\mathrm{CoO}$ ) achieved subgrain formation at lower temperatures and pressures than their alloyed counterparts (such as $\mathrm{KCl}-\mathrm{KBr}, \mathrm{KCl}-\mathrm{NaCl}$, and impure $\mathrm{CoO})$. [35-37, 42]. Specifically in the case of $\mathrm{CoO}$, the stress exponent decreased from $n=4.6$ to $n=3.25$ with the addition of just $0.5 \%$ impurities [42]. These studies indicate that although some pure ceramics have high stress exponents and display Class I behavior, it is possible for impurities to reduce the stress exponents to a regime in which materials display Class II behavior [35-37, 42].

Specifically, Fig. 1 unambiguously illustrates the addition of $2 \%$ aliovalent cation impurities alters the prevalence of subgrains and LAGBs in the final sintered material. The exact mechanism or combination of mechanisms is unknown, but we posit a couple of possibilities to motivate future studies of this phenomenon. One possible explanation for the change in LAGB density is that adding impurities reduces the dislocation mobility during SPS, in some cases to the point where dislocations cannot coalesce to form LAGBs. When dislocation glide is the dominant mechanism, dislocation mobility is impeded by the dragging of solute ion atmospheres, and the resultant steady-state creep rate is inversely proportional to the square of the mismatch in cation size $\left(\dot{\varepsilon} \propto \frac{1}{e^{2}}\right)$ [34]. In other words, a large cation mismatch (e, which is related to strain) will result in a decrease in the dislocation creep rate $(\dot{\varepsilon})$ and an increase in the time needed to form LAGBs. Assuming the reduction in creep rate is based solely on the cation size deviation from $\mathrm{Pb}^{2+}$, we expect to see increasing creep resistance following the trend: $\mathrm{Ag}^{+}<\mathrm{Na}^{+}<\mathrm{K}^{+}$. This is because the cation radius of $\mathrm{Pb}^{2+}$ is $119 \mathrm{pm}$, while the radii for the $\mathrm{Ag}^{+}, \mathrm{Na}^{+}$, and $\mathrm{K}^{+}$cations are $115 \mathrm{pm}, 102 \mathrm{pm}$, and $138 \mathrm{pm}$ (resulting in radial deviations of $-4 \mathrm{pm},-17 \mathrm{pm}$, and $19 \mathrm{pm}$, respectively). Furthermore, although the differences in cation radii of $\mathrm{Na}^{+}$and $\mathrm{K}^{+}$from $\mathrm{Pb}^{2+}$ are $-17 \mathrm{pm}$ and $19 \mathrm{pm}$, respectively, the change in volume of a crystal due to the substitution of a smaller cation is less than the change in volume produced by substituting larger cation with the same deviation in size from the original cation [43]. This difference in lattice strain can help explain the difference in LAGB density between $\mathrm{Na}$ - and $\mathrm{K}$-doped $\mathrm{PbSe}$ despite similar deviations of these substitutional cations from the cation radius of $\mathrm{Pb}^{2+}$.

The behavior of Ag-doped PbSe, however, does not follow the above trend based on cation size difference alone. One consideration we did not account for was that our DFT study indicates that $\mathrm{Ag}^{+}$forms interstitial defects at high levels of doping, while $\mathrm{Na}^{+}$and $\mathrm{K}^{+}$sit on cation sites. Because $\mathrm{Ag}^{+}$is large for an interstitial cation, it will result in much more strain in the lattice than if it was a on a cation site. Therefore, we expect increasing lattice strain from each dopant in the order $\mathrm{Na}^{+}$, followed by $\mathrm{K}^{+}$, followed by $\mathrm{Ag}^{+}$. Additionally, because Ag-doped $\mathrm{PbSe}$ has been reported to have precipitates at concentrations above $1 \%$ [10], we feel the possibility of precipitates should be addressed (despite our PXRD indicating that all samples were single phase $\mathrm{PbSe}$ ). If precipitates were to form in $\mathrm{PbSe}-2 \% \mathrm{Ag}$, these could impede dislocation mobility as well, slowing LAGB formation. However, whether dislocation mobility is inhibited by interstitials or precipitates (or both), it can be hypothesized that the presence of Ag results in atomic and nanoscale structures and defects that impede the mobility of dislocations and prevent LAGBs from forming.

Another explanation for the variation in LAGB density is that stress relaxation mechanisms (such as recovery through polygonization and recrystallization) occur concurrently with deformation during high-temperature creep [32, 44]. During hot plastic deformation, strain softening can occur in parallel with deformation and results in the reduction and redistribution of dislocation density, as well as the potential nucleation and growth of stress free grains through the migration of HAGBs in what is known as dynamic recrystallization. It has also been documented that in addition to processing parameters such as temperature, degree of deformation, and deformation rate, the recrystallization process can be influenced by material properties such as stacking fault energy, second phase particles, and the presence of foreign atoms [32]. Because of the dependence of recrystallization on atomic and nanoscale structures, it is feasible that the variation in dopants (and the defects that they engender) may have an influence over the recrystallization rate in doped $\mathrm{PbSe}$. As a result, some materials may fully undergo dynamic recrystallization or polygonization during SPS while others do not, which would result in varying degrees of subgrain formation, like those we observe.

In summary, we confirmed that creep occurs in PbSe during SPS, and that the identity of dopants added to PbSe influences the subgrain structure in the final material. Furthermore, we 
hypothesize that both dynamic recrystallization and changes in dislocation mobility could contribute to the final microstructure of $\mathrm{PbSe}$, but acknowledge that the exact relationship between these two mechanisms is not yet perfectly understood. To fully understand the relationship between dopants, point defects, and post-processed material microstructure, more in-depth creep studies need to be performed on doped semiconducting materials. With improved knowledge of these mechanisms, informed modification of sintering conditions (such as time, temperature, and pressure) based on dopant species and material composition could lead to better control over final material microstructure, which is vital to optimizing thermoelectric performance.

\section{Grain size}

Designing a thermoelectric material with a tailored grain size is important for a variety of reasons. First, minimizing grain size is beneficial for reducing lattice thermal conductivity because the increase in the number of grain boundaries assists in the scattering of large wavelength phonons $[6,45,46]$. However, recent findings show that in more ionic material systems with low dielectric constants, including some heavily alloyed forms of $\mathrm{PbSe}$, grain boundaries are capable of creating a charge carrier screening effect that is detrimental to low-temperature electrical conductivity [20]. Therefore, when optimizing grain structure in materials that are known to be more ionic, larger grains may actually result in an improvement in efficiency in low-temperature regimes. One such recent example is $\mathrm{Mg}_{3} \mathrm{Sb}_{2}$, in which it was recently found that larger grains resulted in higher overall thermoelectric efficiency [47]. These works emphasize the importance of considering the impact of microstructure on both phonon scattering and charge carrier mobility when engineering thermoelectric materials.

In addition to optimizing transport properties, grain size may also affect a material's sublimation rate at elevated operating temperatures, due to the presence of grain boundaries at the surface of a material. Grain boundaries are known to be sources of elemental segregation, dislocations, and changes in surface topology, all of which may increase sublimation mass loss [22-24]. With these considerations in mind, it is clear that thermoelectric materials should be designed with specific grain sizes selected to achieve both optimal efficiency and reduced material degradation. In this study, we provide evidence that hints at the fact that changes in post-synthetic grain size could be due in part to changes in doping species; however, these changes in grain size could be the result of a variety of processing factors in addition to composition, and a further analysis would have to be performed to establish the relationship between doping, processing, and grain size. However, in the discussion below we do offer one potential reason for grain size variation in our samples as well as describe why double sieving was useful in improving grain size uniformity in this case.

We propose that one reason for our observation of various grain sizes in differently doped samples may be due to a combination of changes in mechanical properties of the doped samples as well as the fact that we prepared precursor powders by crushing and hand grinding ingot material. The solution hardening rate in ionic compounds can be increased by an order of magnitude by introducing aliovalent cations into a material rather than isovalent cations, and it is widely accepted that this is caused by the compensating point defects that form in conjunction with the aliovalent cations [48]. This is confirmed by our own DFT results which reveal that different dopants result in different combinations of point defects and compensating defects. As a result, it should be expected that some PbSe samples in this investigation would be more robust or more brittle than their alternately doped counterparts (which we qualitatively observed while hand grinding), ultimately yielding differences in the distribution of powder particle size after grinding. This potential difference in mechanical properties further illustrates why adding an additional step to the sieving process may have been beneficial to achieving doped $\mathrm{PbSe}$ with a more uniform grain size across materials doped with different dopant species.

\section{High-temperature mass loss due to sublimation}

Sublimation is a major source of degradation in thermoelectric devices which can cause cross-sectional reduction of the thermoelements and can eventually lead to mechanical failure of device components [5]. Because thermoelectric modules should ideally operate for years at elevated temperatures, the materials must be robust to sublimation over extended periods of time. Despite the risk that sublimation poses, it is often overlooked as it is not easily identifiable during transport measurements. This is because when obtaining transport data, thermal cycling lasts for a relatively short period of time, resulting in only minor sublimation. The amount of material that is lost is often not enough to manifest itself as an artifact in the transport data. In addition, if sublimation does take place, the surface pores and pock marks that form are on the order of microns, which is too small to be observed with the naked eye, and too large to be very noticeable when performing TEM or other high resolution with limited fields of view. In $\mathrm{PbSe}$ specifically, $\mathrm{PbSe}(\mathrm{g})$ is the dominant gaseous species, leading us to believe that high-temperature mass loss is due to sublimation of the parent phase, and not necessarily due to the sublimation of the dopant or a second phase [49].

By utilizing the versatility of the SEM, we used a combination of imaging and EBSD to assess the importance of microstructure in facilitating mass loss. Imaging and EBSD both reveal that pores form at grain boundaries upon exposure to temperatures above $600^{\circ} \mathrm{C}$. Additionally, EBSD in Fig. 2 shows 
pores form in the presence of both LAGBs and HAGBs, and further reveals that HAGBs are often the nucleation sites for pore formation, but the pores then have a tendency to grow along LAGBs and HAGBs as sublimation continues.

Pure PbSe has on average the least mass loss compared to the samples with p-type dopants added (see Fig. 4a). This occurs despite pure $\mathrm{PbSe}$ having the smallest average grain size (see Fig. 1) and therefore the least favorable microstructure for preventing sublimation. So, despite having more high-energy defects at its surface, pure PbSe still lost less mass on average during annealing than any of the doped samples. Unlike the pure PbSe sample, the doped samples experience decreasing average mass loss as dopants change from $\mathrm{Na}$, to $\mathrm{K}$, to Ag. This trend in mass loss also corresponds to increasing grain size in the doped samples, and therefore the mass loss of these samples cannot be directly compared without a standardized microstructure.

Although samples were doped with the same nominal amount of dopant, we know that the carrier concentration of these samples can vary by as much as an order of magnitude (as seen in Fig. 6) $[10,11,15]$. The variation in doping efficiency brings into question whether the absolute amount of dopant incorporated into a material's lattice structure affects the sublimation. In other words, we seek to discover whether it is more beneficial to dope a material using a small amount of an efficient dopant rather than using a large amount of a less efficient dopant. To deconvolute both the effect of grain size and test the effect of dopant species and concentration on sublimation, large-grained $\mathrm{PbSe}-2 \% \mathrm{Na}$ and large-grained $\mathrm{PbSe}-0.4 \% \mathrm{Na}$ were synthesized using the "double sieving" method. The lower concentration of $\mathrm{Na}$ was selected to be $0.4 \%$ to provide approximately the same carrier concentration as $2 \% \mathrm{Ag}$ in $\mathrm{PbSe}$ based on the respective doping efficiencies. Sublimation mass loss in Fig. $4 \mathrm{~b}$ shows there is a sizeable reduction in mass loss when the amount of Na-doping is reduced from $2 \%$ to $0.4 \%$. Despite this reduction, the mass loss in the large-grained $\mathrm{PbSe}-0.4 \% \mathrm{Na}$ samples is still comparable to $\mathrm{PbSe}-2 \% \mathrm{Ag}$. Moreover, pure $\mathrm{PbSe}$ appears to be more stable than any of the doped compounds. These results therefore indicate that adding less total dopant to a material is beneficial for reducing mass loss.

It should be noted that we still cannot draw any conclusions about the relative sublimation rates of $\mathrm{PbSe}$ containing different dopants due to several factors. The first is that even when adjusting our synthesis, there is still a variation in LAGBs population, and this can contribute to sublimation differences. Although we observe that the LAGBs are not nearly as volatile as HAGBs (which can be seen in Fig. 2b and d), it is still a concerning difference in microstructure that must be accounted for. Secondly, because of the error in mass measurements discussed earlier (see SI, Table SI), the average sublimation rates of large-grained $\mathrm{PbSe}-0.4 \% \mathrm{Na}$ and $\mathrm{PbSe}-2 \% \mathrm{Ag}$ are too similar to derive any meaningful conclusions concerning the difference in sublimation rate due to dopant species. Despite these limitations, the sublimation experiments still suggest that the sublimation of PbSe increases due to the presence p-type dopants, and that the concentration of dopant added affects the thermal stability of doped $\mathrm{PbSe}$ compounds. This study also provides further evidence of the influence of microstructure on sublimation and shows why understanding the effects of dopants on microstructure is necessary for producing more robust thermoelectric materials.

\section{Summary and conclusions}

In this study, we used PbSe to emphasize the care that should be taken when selecting a dopant to use in a thermoelectric material. Conventional practice utilizes dopants primarily for the improvement of carrier concentration while providing the additional benefit of thermal conductivity reduction through point defect scattering. This work investigates alternative considerations to conventional doping practice and seeks to motivate further investigation into the wider implications of doping on material microstructure and properties. Using DFT we illustrate how the introduction of different dopant species changes the dominant type of underlying point defects, and we confirmed the presence of the specific point defect species predicted by DFT by comparing these results to established trends in doping efficiency. We also offer discussions on how these same point defects may alter the mechanical properties (through aliovalent solution hardening and dislocation mobility reduction). Furthermore, we demonstrate how using this information to implement intentional processing techniques (such as "double sieving" or altering SPS processing conditions) can result in a desired microstructure that enables a material to achieve an optimal combination of thermoelectric efficiency and thermal stability. In conclusion, we propose that in order to achieve true material optimization, it is essential to understand the broader implications of selecting particular dopants for a thermoelectric material system, because dopants can alter material microstructure, which subsequently affects overall thermoelectric performance. We hope that this work inspires future investigations on the effects of dopants at elevated temperatures on the microstructure and properties of semiconducting materials during both processing and high-temperature operation.

\section{Methods}

\section{DFT calculations}

Our calculations were based on density functional theory (DFT) within the generalized-gradient approximation (GGA) [50]. The projector augmented wave pseudopotential was applied as implemented in the VASP package [51]. Calculations for bulk $\mathrm{PbSe}$ with different defects were performed using a $3 \times 3 \times 3$ for 
54 atom cell with very dense k-point mesh corresponding to $4000 \mathrm{k}$-points per reciprocal atom. The plane-wave basis-set cutoff energy was set to $500 \mathrm{eV}$ and the convergence criterion with respect to self-consistent iterations was assumed the residual forces were less than $0.01 \mathrm{eV} / \mathrm{A}$. In the defect calculations, the lattice parameters were fixed to the calculated bulk values, but all the internal coordinates were fully relaxed.

The formation energy of a defect $X$ in charge state $q$ was defined as

$$
E^{f}\left(X^{q}\right)=E_{\text {tot }}\left(X^{q}\right)-E_{\text {tot }}^{\text {bulk }}-\Delta n_{i} \mu_{i}+q\left(E_{V}+\Delta_{V}+\varepsilon_{F}\right)
$$

in such a way that the negative values suggest favorable formation and the positive values unfavorable [52]. In the equation, $E_{\text {tot }}\left(X^{q}\right)$ and $E_{\text {tot }}^{\text {bulk }}$ are, respectively, the total energies of a supercell containing the defect $X$ and of a supercell of the perfect bulk material; $\mu_{i}$ is the atomic chemical potential of species $i$ (and is referenced to the standard state), and $\Delta n_{i}$ denotes the number of atoms of species $i$ that have been added $\left(\Delta n_{i}>0\right)$ or removed $\left(\Delta n_{i}<0\right)$ to form the defect. $\varepsilon_{F}$ is the Fermi level referenced to the valence-band maximum in the bulk $\left(E_{V}\right)$. The potential alignment term $\Delta_{V}$ is the shift in the band positions due to the presence of the charged defect and the neutralizing background, obtained by aligning the average electrostatic potential in regions far away from the defect to the bulk value. Note that we denote defect $\mathrm{X}$ in charge state $\mathrm{q}$ as $X^{q}$. The chemical potential selection in the above defect formation energy calculations is based on the phase diagrams of A-Pb-Se $(\mathrm{A}=\mathrm{Na}, \mathrm{K}, \mathrm{Ag})$ from the open quantum materials database $[53,54]$. In each phase diagram, the convex hulls have been constructed from a set of possible phases using grand canonical linear program methods. The chemical potentials corresponding to each set of the threephase equilibrium are solved relative to the composition of each element. The nominal composition of doped $\mathrm{PbSe}$ in this investigation is $\mathrm{A}_{\mathrm{x}} \mathrm{Pb}_{1-\mathrm{x}} \mathrm{Se}$, and can alternatively be written as $\mathrm{xASe}-$ (1-x)PbSe. Therefore, the chemical potentials used in the DFT calculations plotted in Fig. 5 were obtained from the equilibrium three-phase regions in the $\mathrm{A}-\mathrm{Pb}-\mathrm{Se}$ phase diagram which either encompass or include the $\mathrm{ASe}-\mathrm{PbSe}$ tie-line. These regions are $\mathrm{PbSe}-\mathrm{NaSe}-\mathrm{Na} 2 \mathrm{Se}$, PbSe-KSe-K2Se, and PbSe-Se-Ag2Se for the Na-doped, K-doped, and Ag-doped defect energy calculations, respectively. Additional defect energies calculated using various chemical potentials can be found in the SI in Table SII.

\section{Material synthesis}

\section{Synthesis}

The starting materials were $\mathrm{Pb}$ wire $(99.99 \%$, American Elements), Se shot (99.99\%, American Elements), Na cubes (99.95\%, Sigma Aldrich), Ag pieces (99.999\%, Sigma Aldrich), and K cubes (99.95\%, Sigma Aldrich). Polycrystalline ingots of doped PbSe were first synthesized as follows. According to the desired nominal compositions, $\mathrm{A}_{\mathrm{x}} \mathrm{Pb}_{1-\mathrm{x}} \mathrm{Se}(\mathrm{A}=\mathrm{Na}, \mathrm{Ag}, \mathrm{K})$ stoichiometric quantities of each element were weighed into 13-mm-diameter carbon-coated fused silica tubes and then flame sealed at $\sim 2 \times 10^{-3}$ Torr. Typical samples used $10 \mathrm{~g}$ of total starting material. The tubes were heated in a box furnace to $500{ }^{\circ} \mathrm{C}$ over the course of $12 \mathrm{~h}$, held for $2 \mathrm{~h}$, and then heated to $1150{ }^{\circ} \mathrm{C}$ in $7 \mathrm{~h}$ where they dwelled at temperature for $5 \mathrm{~h}$. The tubes were next brought to room temperature over $12 \mathrm{~h}$.

\section{Post-synthetic processing}

The as-cast ingots were removed from the tubes and subject to different preparatory procedures. All samples were hand ground into powder with an agate mortar and pestle, and the sample powders were then passed through a sieve using either a 53, 70, or $150 \mu \mathrm{m}$ mesh. The use of different mesh sizes allows for control over the grain size in the final samples. The sieved powder was loaded into a $12.7 \mathrm{~mm}$ graphite and sintered into dense pellets by Spark Plasma Sintering (SPS-211LX, Fuji Electronic Industrial Co. Ltd). The sintering process was conducted under dynamic vacuum at $550{ }^{\circ} \mathrm{C}$, and the samples were held at temperature for $10 \mathrm{~min}$ under $40 \mathrm{MPa}$ of uniaxial pressure before being cooled to room temperature. Square prisms with approximate dimensions of $4 \times 4 \times 2 \mathrm{~mm}^{3}$ were cut from roughly the same location of their respective pellets with the large face perpendicular to the pressing direction for sample analysis.

\section{Electron microscopy sample preparation}

Surface scratches and deformation are detrimental to Electron Backscattered Diffraction (EBSD) because this technique uses a Scanning Electron Microscope (SEM) to collect diffraction patterns to obtain crystallographic information from the surface of a sample. Because of this technique's reliance on diffraction, any deviations from the ideal structure in the surface results in a distorted signal [55]. As a result, careful polishing was required to eliminate surface deformation as much as possible so that surface micro-strain did not obscure the true crystal structure of the sample.

The cut samples were cold mounted into quick dry epoxy, with the large face exposed. The sample was then ground using 600,800 , and 1200 grit $\mathrm{SiC}$ grinding paper on a rotary grinding wheel, using ethanol as a water-free lubricant. Next samples were polished for $30 \mathrm{~min}$ and $45 \mathrm{~min}$ with $1 \mu \mathrm{m}$ and $0.1 \mu \mathrm{m}$ glycolbased diamond slurry, respectively. Upon finishing this step, samples were placed into a vibratory polisher for $3 \mathrm{~h}$ at $62.7 \mathrm{~Hz}$ in a bath of $0.1 \mu \mathrm{m}$ glycol-based diamond slurry. The samples were then removed from the cold mount using acetone, flipped 
over, and this process was repeated so that both large faces had the same polish and surface roughness.

\section{Scanning electron microscopy}

After polishing, samples were mounted to SEM stubs using paraffin wax and then copper tape was applied as a contact between the sample and the SEM stub to ensure that there was an electrically conductive connection. Any sample charging in the SEM during EBSD can result in sample drift and other charging effects that interfere with image and data collection. Therefore, chamber pressure was maintained at 0.1 Torr to further reduce charging effects [56]. For both imaging and EBSD, accelerating voltage was set to $30 \mathrm{kV}$ with a working distance of approximately $10 \mathrm{~mm}$ and was carried out using a FEI Quanta 650 ESEM. EBSD patterns were collected over an area of $200 \mu \mathrm{m} \times 200 \mu \mathrm{m}$ with a $0.8 \mu \mathrm{m}$ step size using an Oxford Instrument's Nordlys II detector and post-processing was performed using Oxford Instruments CHANNEL 5 software suite. After thermal treatment, samples did not need to be re-polished. In fact, due to annealing, the surface deformations that remained after polishing were reduced yielding sharper EBSPs. Consequently, thermally treated samples were immediately characterized under the same SEM conditions described above without any additional grinding or polishing.

\section{Powder X-ray diffraction}

To determine the phase of our synthesized materials, we collected powder X-ray diffraction (PXRD) measurements on a Rigaku Miniflex 600 instrument using $\mathrm{Cu}$ Ka radiation with wavelength $\lambda=1.5406 \AA$ and operating at $40 \mathrm{kV}$ and $15 \mathrm{~mA}$ and with a $K \beta$ filter. The measurements were performed on SPSsintered samples, and the powder patterns are shown in SI Fig. S6. All patterns show good agreement with the expected PbSe structure with no evidence for secondary phases, indicating successful preparation of the desired compounds.

\section{Thermal treatment and mass loss measurements}

After preliminary SEM imaging and EBSD had been performed, samples were weighed and then flame sealed in $9 \mathrm{~mm}$ diameter tubes at $\sim 2 \times 10^{-3}$ Torr. The tubes were then placed in furnaces pre-heated to $650^{\circ} \mathrm{C}$ and annealed for $48 \mathrm{~h}$. After dwelling, the samples were quenched in air. The samples were removed from the tubes and weighed again to determine their mass loss during annealing. It should be noted that hightemperature mass loss experiments resulted in large error due to differences in surface quality around edges, cracking of samples while annealing, and potential chipping of brittle samples throughout the annealing and measurement process. Because of the possibility for large amounts of error, 3 to 5 trials were performed for each sample to obtain average mass loss measurements at elevated temperatures. Yet even with these precautions, doped samples had standard deviations as high as $3 \%$ mass loss, while the standard deviation of pure $\mathrm{PbSe}$ remained at around $1 \%$.

\section{Acknowledgments}

The authors would like to thank Professor David Dunand for taking the time to provide useful discussions on the creep of ceramic materials. This work is primarily supported by the U.S Department of Energy, Office of Science and Office of Basic Energy Sciences under award number DE-SC0014520. This work made use of the EPIC facility of Northwestern University's NUANCE Center, which has received support from the Soft and Hybrid Nanotechnology Experimental (SHyNE) Resource (NSF ECCS-1542205); the MRSEC program (NSF DMR-1720139) at the Materials Research Center; the International Institute for Nanotechnology (IIN); the Keck Foundation; and the State of Illinois, through the IIN. This work also made use of the MatCI Facility which receives support from the MRSEC Program (NSF DMR-1720139) of the Materials Research Center at Northwestern University. This material is based upon work supported by the National Science Foundation Graduate Research Fellowship under Grant No. DGE1324585. Portions of this work were previously published in the dissertation of Jann Albert Grovogui ("Improving Thermoelectric Performance Through Defect Engineering with an Emphasis on the Mesoscale", which was defended on October $19,2020)$ and are reproduced (adapted) with permission from Jann Albert Grovogui.

\section{Funding}

This work is primarily supported by the U.S Department of Energy, Office of Science and Office of Basic Energy Sciences under award number DE-SC0014520, and is additionally supported by the National Science Foundation Graduate Research Fellowship under Grant No. DGE-1324585.

\section{Data availability}

The datasets generated during and/or analyzed during the current study are available from the corresponding author on reasonable request.

\section{Compliance with ethical standards}

Conflict of interest On behalf of all authors, the corresponding author states that there is no conflict of interest. 


\section{Open Access}

This article is licensed under a Creative Commons Attribution 4.0 International License, which permits use, sharing, adaptation, distribution and reproduction in any medium or format, as long as you give appropriate credit to the original author(s) and the source, provide a link to the Creative Commons licence, and indicate if changes were made. The images or other third party material in this article are included in the article's Creative Commons licence, unless indicated otherwise in a credit line to the material. If material is not included in the article's Creative Commons licence and your intended use is not permitted by statutory regulation or exceeds the permitted use, you will need to obtain permission directly from the copyright holder. To view a copy of this licence, visit http://creativecommons.org/ licenses/by/4.0/.

\section{Supplementary Information}

The online version of this article (https://doi.org/10.1557/ s43578-021-00130-8) contains supplementary material, which is available to authorized users.

\section{References}

1. G.J. Snyder, E.S. Toberer, Complex thermoelectric materials. Nat. Mater. 7(2), 105 (2008)

2. J. He, M.G. Kanatzidis, V.P. Dravid, High performance bulk thermoelectrics via a panoscopic approach. Mater. Today 16(5), 166 (2013)

3. S.B. Riffat, X. Ma, Thermoelectrics: a review of present and potential applications. Appl. Therm. Eng. 23(8), 913 (2003)

4. M. Hamid Elsheikh, D.A. Shnawah, M.F.M. Sabri, S.B.M. Said, M. Haji Hassan, M.B. Ali Bashir, M. Mohamad, A review on thermoelectric renewable energy: principle parameters that affect their performance. Renew. Sustain. Energy Rev. 30, 337 (2014)

5. J. Yang, T. Caillat, Thermoelectric Materials for Space. MRS Bull. 31(March), 224 (2006)

6. K. Biswas, J. He, I.D. Blum, C.I. Wu, T.P. Hogan, D.N. Seidman, V.P. Dravid, M.G. Kanatzidis, High-performance bulk thermoelectrics with all-scale hierarchical architectures. Nature 489(7416), 414 (2012)

7. L.M. Daniels, S.N. Savvin, M.J. Pitcher, M.S. Dyer, J.B. Claridge, S. Ling, B. Slater, F. Corà, J. Alaria, M.J. Rosseinsky, Phonon-glass electron-crystal behaviour by A site disorder in n-type thermoelectric oxides. Energy Environ. Sci. 10(9), 1917 (2017)

8. H. Alam, S. Ramakrishna, A review on the enhancement of figure of merit from bulk to nano-thermoelectric materials. Nano Energy 2(2), 190 (2013)
9. D. An, S. Chen, Z. Lu, R. Li, W. Chen, W. Fan, W. Wang, Y. Wu, Low thermal conductivity and optimized thermoelectric properties of p-type Te-Sb2Se3: synergistic effect of doping and defect engineering. ACS Appl. Mater. Interfaces 11(31), 27788 (2019)

10. S. Wang, G. Zheng, T. Luo, X. She, H. Li, X. Tang, Exploring the doping effects of $\mathrm{Ag}$ in p-type PbSe compounds with enhanced thermoelectric performance. J. Phys. D 44(47), 475304 (2011)

11. H. Wang, Y. Pei, A.D. Lalonde, G.J. Snyder, Heavily doped p-type PbSe with high thermoelectric performance: an alternative for PbTe. Adv. Mater. 23(11), 1366 (2011)

12. Q. Zhang, H. Wang, W. Liu, H. Wang, B. Yu, Q. Zhang, Z. Tian, G. Ni, S. Lee, K. Esfarjani, G. Chen, Z. Ren, Enhancement of thermoelectric figure-of-merit by resonant states of aluminium doping in lead selenide. Energy Environ. Sci. 5(1), 5246 (2012)

13. Y. Pei, H. Wang, G.J. Snyder, Band engineering of thermoelectric materials. Adv. Mater. 24(46), 6125 (2012)

14. Y. Yu, M. Cagnoni, O. Cojocaru-Mirédin, M. Wuttig, Chalcogenide thermoelectrics empowered by an unconventional bonding mechanism. Adv. Funct. Mater. 30(8) (2020)

15. Q. Zhang, F. Cao, W. Liu, K. Lukas, B. Yu, S. Chen, C. Opeil, D. Broido, G. Chen, Z. Ren, Heavy doping and band engineering by potassium to improve the thermoelectric figure of merit in p-type $\mathrm{PbTe}, \mathrm{PbSe}$, and PbTe 1- ySe y. J. Am. Chem. Soc. 134(24), 10031 (2012)

16. A.J. Minnich, M.S. Dresselhaus, Z.F. Ren, G. Chen, Bulk nanostructured thermoelectric materials: current research and future prospects. Energy Environ. Sci. 2(5), 466 (2009)

17. J.H. Son, M.W. Oh, B.S. Kim, S.D. Park, B.K. Min, M.H. Kim, H.W. Lee, Effect of ball milling time on the thermoelectric properties of p-type (Bi, Sb)2Te3. J. Alloys Compd. 566, 168 (2013)

18. S. LeBlanc, Thermoelectric generators: linking material properties and systems engineering for waste heat recovery applications. Sustain. Mater. Technol. 1-2, 26 (2014)

19. T.J. Slade, J.A. Grovogui, S. Hao, T.P. Bailey, R. Ma, X. Hua, M.G. Kanatzidis, Absence of nanostructuring in $\mathrm{NaPbmSbTem}+2$ : solid solutions with high thermoelectric performance in the intermediate temperature regime. J. Am. Chem. Soc. 140(22), 7021 (2018)

20. T.J. Slade, J.A. Grovogui, J.J. Kuo, S. Anand, T.P. Bailey, M. Wood, C. Uher, G.J. Snyder, V.P. Dravid, M.G. Kanatzidis, Understanding the thermally activated charge transport in $\mathrm{NaPb} \mathrm{m}$ SbQ $\mathrm{m}+2(\mathrm{Q}=\mathrm{S}, \mathrm{Se}, \mathrm{Te})$ thermoelectrics: weak dielectric screening leads to grain boundary dominated charge carrier scattering. Energy Environ. Sci. 13, 1509 (2020)

21. M. Winning, A.D. Rollett, Transition between low and high angle grain boundaries. Acta Mater. 53(10), 2901 (2005)

22. D. Zhao, C. Tian, S. Tang, Y. Liu, L. Chen, High temperature oxidation behavior of cobalt triantimonide thermoelectric material. J. Alloys Compd. 504(2), 552 (2010) 
23. Z.A. Munir, J.P. Hirth, Surface morphology of sublimated crystals of cadmium and zinc sulfides. J. Appl. Phys. 41(6), 2697 (1970)

24. W.W. Mullins, Theory of thermal grooving. J. Appl. Phys. 28(3), 333 (1957)

25. R.J.D. Tilley, Defects in Solids (Wiley, Hoboken, 2008).

26. J. Male, M.T. Agne, A. Goyal, S. Anand, I.T. Witting, V. Stevanović, G.J. Snyder, The importance of phase equilibrium for doping efficiency: Iodine doped PbTe. Mater. Horizons 6(7), 1444 (2019)

27. A. Walsh, A. Zunger, Instilling defect tolerance in new compounds. Nat. Mater. 16(10), 964 (2017)

28. W. Walukiewicz, Intrinsic limitations to the doping of wide-gap semiconductors. Physica B 302-303, 123 (2001)

29. S.I. Kim, K.H. Lee, H.A. Mun, H.S. Kim, S.W. Hwang, J.W. Roh, D.J. Yang, W.H. Shin, X.S. Li, Y.H. Lee, G.J. Snyder, S.W. Kim, Dense dislocation arrays embedded in grain boundaries for high-performance bulk thermoelectrics. Science (-80) 348(6230), 109 (2015)

30. Y. Pan, U. Aydemir, J.A. Grovogui, I.T. Witting, R. Hanus, Y. $\mathrm{Xu}$, J. Wu, C.F. Wu, F.H. Sun, H.L. Zhuang, J.F. Dong, J.F. Li, V.P. Dravid, G.J. Snyder, Melt-centrifuged (Bi, Sb)2Te3: engineering microstructure toward high thermoelectric efficiency. Adv. Mater. 30(34), 1802016 (2018)

31. Z. Chen, B. Ge, W. Li, S. Lin, J. Shen, Y. Chang, R. Hanus, G.J. Snyder, Y. Pei, Vacancy-induced dislocations within grains for high-performance PbSe thermoelectrics. Nat. Commun. 8, 1 (2017)

32. K.K. Alaneme, E.A. Okotete, Recrystallization mechanisms and microstructure development in emerging metallic materials: a review. J. Sci. Adv. Mater. Dev. 4(1), 19 (2019)

33. G.S. Rohrer, Grain boundary energy anisotropy: a review. J. Mater. Sci. 46(18), 5881 (2011)

34. W.R. Cannon, T.G. Langdon, Creep of ceramics-Part 2 An examination of flow mechanisms. J. Mater. Sci. 23(1), 1 (1988)

35. H. Duong, M. Beeman, J. Wolfenstine, High temperature creep behavior and substructure in $\mathrm{KCl}-\mathrm{KBr}$ solid solution alloys. Acta Metall. Mater. 42(3), 1001 (1994)

36. F.A. Mohamed, T.G. Langdon, Creep behavior of ceramic solidsolution alloys. J. Am. Ceram. Soc. 58(11), 533 (1975)

37. W.R. Cannon, O.D. Sherby, High-temperature creep of $\mathrm{NaCl}-\mathrm{KCl}$ solid-solution alloys. J. Am. Ceram. Soc. 53(6), 346 (1970)

38. Z.P. Guan, D.C. Dunand, Compressive creep behavior of cast Bi2Te3. Mater. Sci. Eng. A 565, 321 (2013)

39. M.C. Chang, M.T. Agne, R.A. Michi, D.C. Dunand, G.J. Snyder, Compressive creep behavior of hot-pressed GeTe based TAGS-85 and effect of creep on thermoelectric properties. Acta Mater. 158, $239(2018)$
40. C.C. Li, G.J. Snyder, D.C. Dunand, Compressive creep behaviour of hot-pressed PbTe. Scr. Mater. 134, 71 (2017)

41. W.R. Cannon, O.D. Sherby, Third-power stress dependence in creep of polycrystalline nonmetals. J. Am. Ceram. Soc. 56(3), 157 (1973)

42. V. Krishnamachari, J.T. Jones, Compressive creep of CoO single crystals. J. Am. Ceram. Soc. 57(11), 506 (1974)

43. S.T. Murphy, H. Lu, R.W. Grimes, General relationships for isovalent cation substitution into oxides with the rocksalt structure. J. Phys. Chem. Solids 71(5), 735 (2010)

44. E. Nes, Recovery revisited. Acta Metall. Mater. 43(6), 2189 (1995)

45. P.G. Klemens, Phonon scattering and thermal resistance due to grain boundaries. Int. J. Thermophys. 15(6), 1345 (1994)

46. Z. Wang, J.E. Alaniz, W. Jang, J.E. Garay, C. Dames, Thermal conductivity of nanocrystalline silicon: importance of grain size and frequency-dependent mean free paths. Nano Lett. 11(6), $2206(2011)$

47. J.J. Kuo, S.D. Kang, K. Imasato, H. Tamaki, S. Ohno, T. Kanno, G.J. Snyder, Grain boundary dominated charge transport in Mg3Sb2-based compounds. Energy Environ. Sci. 11(2), 429 (2018)

48. T.E. Mitchell, A.H. Heuer, Solution hardening by aliovalent cations in ionic crystals. Mater. Sci. Eng. 28(81), 81 (1977)

49. E.E. Hansen, Z.A. Munir, M.J. Mitchell, Sublimation pressure and sublimation coefficient of single-crystal lead selenide. J. Am. Ceram. Soc. 52(11), 610 (1969)

50. J.P. Perdew, K. Burke, M. Ernzerhof, Generalized gradient approximation made simple. Phys. Rev. Lett. 77(18), 3865 (1996)

51. G. Kresse, J. Furthmüller, Efficient iterative schemes for ab initio total-energy calculations using a plane-wave basis set. Phys. Rev. B 54(16), 11169 (1996)

52. A. Janotti, C.G. Van De Walle, Native point defects in $\mathrm{ZnO}$. Phys. Rev. B 76(16), 1 (2007)

53. J.W. Doak, K.J. Michel, C. Wolverton, Determining dilute-limit solvus boundaries in multi-component systems using defect energetics: $\mathrm{Na}$ in PbTe and PbS. J. Mater. Chem. C 3, 10630 (2015)

54. J.E. Saal, S. Kirklin, M. Aykol, B. Meredig, C. Wolverton, Materials design and discovery with high-throughput density functional theory: the Open Quantum Materials Database (OQMD). JOM 65, 1501 (2013)

55. G.L. Wynick, C.J. Boehlert, Use of electropolishing for enhanced metallic specimen preparation for electron backscatter diffraction analysis. Mater. Charact. 55(3), 190 (2005)

56. A. Mohan, N. Khanna, J. Hwu, D.C. Joy, Secondary contrast in the variable pressure scanning electron microscope. Scanning 19(3), 221 (1997) 